\title{
Pioneer and Complementary Research: The True Research Taxonomy
}

\author{
Bennie Berkeley \\ University of the West Indies, St. Augustine, Trinidad \\ Email: Bennie.Berkeley@sta.uwi.edu
}

Received 3 November 2014; revised 15 December 2014; accepted 4 January 2015

Copyright (C) 2015 by author and OALib.

This work is licensed under the Creative Commons Attribution International License (CC BY). http://creativecommons.org/licenses/by/4.0/

(c) (i) Open Access

\section{Abstract}

This position paper proposes that the terms pioneer and complementary are better suited to the classification of research on grounds that they provide the best answer to the epistemological question: what is the purpose of research? Alternatively it purports that quantitative and qualitative are better used to describe types of data. It is the position of this paper that research lies along a continuum polarized by quantitative (positivistic) at one end and qualitative (constructivist) at the other. Multi-methods including mixed and non-mixed designs lie along this continuum. Also it proposes that pioneer and complementary are more appropriate descriptors of research as they are capable of drawing attention to the major significance of scientific inquiry in social development.

\section{Keywords}

Complementary, Data, Research, Pioneer

Subject Areas: Philosophy, Sociology

\section{Introduction}

The main objective of this paper is to demonstrate that pioneer and complementary are appropriate for describing social research. To accomplish this end it is necessary to propose an alternative epistemological question: what is the purpose of research? The answer to this question disfavors any answer of quantitative research or qualitative research and favors pioneer and complementary. In addition, I point out some of the limitations in the quantitative-qualitative research typology, seek to distinguish types of research from types of data and finally strengthen the case for the adoption of the pioneer-complementary research typology. One argument is the complementarity principle. It suggests that we can know more about human behavior in successive research inquiries through the unearthing of new knowledge (similar to or different from existing knowledge). The second and minor objective is to suggest that quantitative and qualitative appropriately describe types of data. 
Social scientists have long mulled over the choice of research methods. Some prefer statistics, others rich thick descriptions of phenomena. For me it is time to set the record straight by postulating that the terms quantitative and qualitative cannot always be logically applied to the classification of research. This claim is made on the premise that the most significant epistemological question is: What is the purpose of the research or for what purpose is data being collected? The answer to this question may be to explain a new social phenomenon (pioneer a theory) or add to an already existing theory (complement a theory). To answer this question using the terms quantitative or qualitative would be illogical for the reason that they say nothing about the usefulness of the final knowledge generated by systematic data analysis.

I propose that quantitative and qualitative are inherently bound up with types of data. A researcher can collect quantitative and qualitative data using a single research question without recourse to use of mixed methods. A social scientist wishing to find out the causes of high or low academic attainment can resort to multiple regression analysis of interval or ratio level data such as student grades, GPA and study habits measured by the number of hours of study weekly. Alternatively the same researcher can conduct in-depth interviews of a small sample of students, parents and teachers in an attempt to discover the main factors they attribute for success in academic attainment.

Another argument supporting the need for intellectual liberation from the restrictions imposed by use of the terms quantitative research and qualitative research is presented in the use of a single data collection instrument. Questionnaires can provide closed-ended items for quantitative analysis and open-ended questions for respondents to provide more detailed (qualitative) descriptions of their thoughts on a particular subject. In the latter case the obvious limitation is that the interviewer cannot ask participants (for self-administered instruments) to clarify issues or provide additional details to enhance data validity.

\section{Limitations of Quantitative/Qualitative Research Classification}

By dichotomizing research into quantitative and qualitative it demonstrates an opportunity missed; that is, to view them as complementary. On their own they present partial understandings of social phenomena. For example, does the term quantitative have any independent effect on the reader's understandings of the findings or their purpose? Does it tell the reader whether a researcher has contributed anything to enhance our understanding of a social phenomenon? The same can be said of usage of the term qualitative.

Another point of significance is that describing research as quantitative or qualitative in no way enables appreciation of data analysis, data discussion and presentation. In today's world of research quantitative and qualitative data can produce similar results. The example provided for the study of academic attainment is a case in point. Both can draw attention to the factors associated with student attainment levels. What is more important is that knowledge is systematically collected using valid and reliable quantitative (surveys and statistics) and qualitative methods (interviews and participant observation).

The traditional quantitative-qualitative classification makes the foible of ignoring the concepts of value, process and product. I say this to re-emphasize the fact that in modern production systems products are labeled and packaged to enhance their marketability/value. Why is this not the case in the social sciences? We continue to wrangle and debate about quantitative and qualitative researches, in terms of their appropriateness, without considering their purpose. If we reflect on the origin of sociology we would remediate this proclivity on the premise that sociology was introduced in intellectual and academic discourses for understanding human behavior.

If social scientists reflect carefully on their data collection methods they will come to the indisputable conclusion that the second research stage/process phase is the collection of data. It is here that the terms quantitative and qualitative have great import. Researchers devise data collection instruments that collect one and/or the other data type. It is incomprehensible why we have muddled descriptors of process with those of outcome/product. Failure to separate them has created descriptive anomalies-descriptions incongruent with neither research purpose nor product.

The product (knowledge for social improvement) must not be confused with the raw material (statistics and text). It can never be over-emphasized that it is more worthwhile to consider the process through which data is transformed, shaped and analyzed for it to be of much real value. The analysis of data is so dynamic that any attempt at shedding light on an aspect of social life must be welcomed, encouraged and evaluated in terms of its contribution to social progress and advancement. The simple truth is that quantitative and qualitative are appropriate for describing types of data analysis. 
I do not discredit the fact that some research projects will be palpable only if one or the other data type is employed. However, what I take umbrage to is the way we continue to evaluate research from these pseudo philosophical polarities without considering their contribution to our understanding of social behavior. Empathetically, I proclaim that there can be quantitative and qualitative; data, methods of data collection and methods of data analysis. However, I contest the claim that there is quantitative research and qualitative research once the question is posed; what is the purpose of the research?

\section{Differentiating Types of Research from Types of Data}

I advocate that the terms quantitative and qualitative are best used to describe types of data whether from primary or secondary sources. When issues of purpose, process and outcome are combined the results are undeniably complementary-providing a full and more valid understanding of social life. These three issues can be represented diagrammatically (Figure 1 ).

Figure 1 demonstrates three research issues: purpose, process and outcome. These issues reflect an approach to research which I coin the Purpose, Process and Outcome Approach (PPOA $\left./ \mathrm{P}^{2} \mathrm{OA}\right)$. Within it is the interconnection between research and data. They provide opportunity for me to reaffirm an earlier postulation that quantitative and qualitative are not appropriate descriptors of research type when the research purpose is to gain deep understanding of human behavior/social life by arriving at new knowledge. Quantitative and qualitative are of descriptively impotent constructs of research outcomes. However, the terms pioneer and complementary have the power to inform the reader about the specific purpose of the research.

Pioneer research signifies to readers that the research attempted to fill a knowledge, methodological, theoretical, social science or social gap. A gap exists wherever answers to research questions have been elusive or nonexistent at the time they are posed. Pioneer studies embody the same philosophy as grounded theory of Glasser and Strauss [1], Strauss and Corbin [2]. However, this paper departs from the assumption that only qualitative data is appropriate for use in grounded theory. However, in a later paper I will argue that grounded theory can be developed using any type of data or a combination of quantitative and qualitative. Durkheim's [3] study of suicide indeed is a pioneer and grounded one as he employed principles of induction via comparison of suicide statistics for 11 European societies.

Complementary research makes no specific claim to gap filling. This is its major distinction from pioneer research. Its main purpose to challenge, test or update knowledge. Sociology and other social sciences are replete with rich examples of complementary research. Atkinsons' [4] criticism of Durkheim's analysis of suicide is noteworthy. Instead of examining suicide as social fact, Atkinson proposed that coroners produce suicide cases based on a method Garfinkel [5] described as Documentary-a combination of reflexivity and indexicality. In short it purports that people make assumptions of events based on their experience and the weight of the evidence defined in a specific or particular context. Initially a case of someone found dead in a closed car with a running motor is more likely to be categorized as suicide than someone found dead in a home ransacked and bloody.
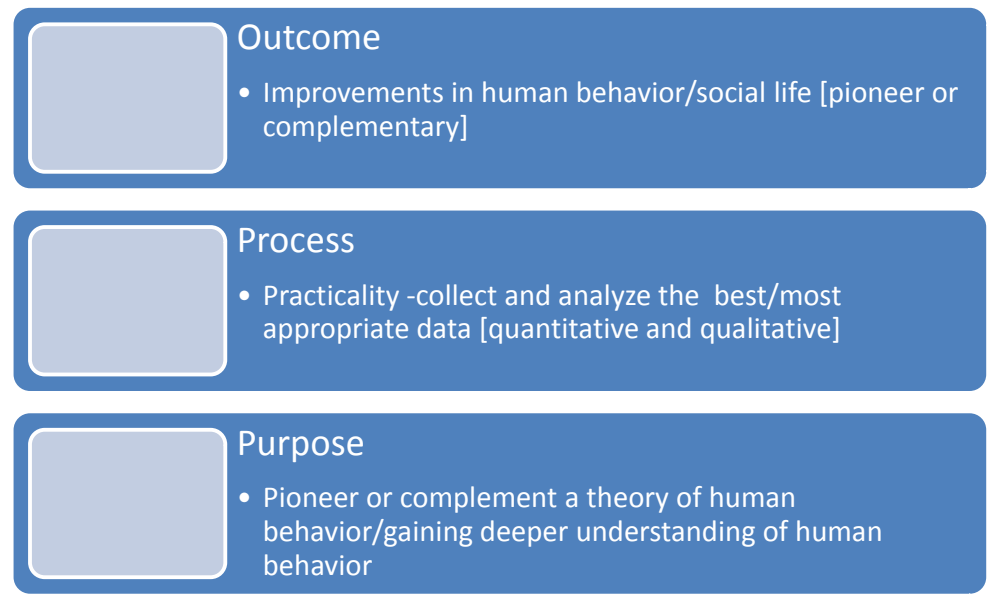

Figure 1. The purpose, process and outcome approach to research 
Once there is compatibility between process and purpose it is fitting that practicality (issues of time, cost, suitability of methods etc.) guide the choice of data. It is in the process phase that the terms quantitative data and qualitative data are of greatest relevance. However, relevance is based on their appropriateness in data description. Indeed it is palpable to distinguish quantitative from qualitative data. The normal distinction quantitative, numerical; qualitative-text based applies.

I make no claim about the comparative advantages of one data type over the other. Their suitability depends too on the research aims and objectives-an argument made in the opening paragraph. There is no foolproof algorithm for data use. It all depends on the "fit" between the data and the research context. A researcher may opt for either quantitative or qualitative alone, a mixture of the two or a multi-approach where no mixing ensues. Garfinkel [5] a proponent of ethnomethodology made extensive use of interviews to understand power holding in conversation interruption between the sexes. It was convenient for him to observe conversations to ascertain which gender was more prone to chart their course.

In evaluating research outcomes the first two phases (purpose and process) must be taken into account. As a general rule the outcome inevitably is to gain a deeper understanding of human behavior/social life in order to improve it. The depth of understanding is evaluated based on the amount and type of data used. Other factors such as time, cost and theoretical conceptualization should be factored in too. For instance, we assume that the longer participants are observed or studied the likelier they are to act naturally giving rise to data validity.

From this standpoint it is unquestionably clear that pioneer and complementary have explanatory power in the description of research. Consequently it is feasible that quantitative and qualitative have greater power in describing data types and data collection methods and data analysis techniques.

\section{Institutionalizing the Pioneer and Complementary Research Typology}

If the question what is the purpose of research is to be answered meaningfully, an appropriate typology is pioneer research-complementary research. Pioneer and complementary produce accounts of social life that are more complete/comprehensive and relevant. Together they explicate the ontological state of being; one in which the knower is better informed yet never has all information about a particular event or occurrence. At best they help us appreciate some features of an event. I conclude that complementary underscores the point that knowledge is relative-it differs according to time, place and context. Information useful for describing behavior in one context, culture (including sub-culture) may not be so relevant in another. Whether research supports or criticizes another it remains complementary in nature.

A good example of complementarity/knowledge relativity is provided in Oakley's [6] critique of Murdock's [7] assessment of the 'fit' between biology and the domestic division of labor. Murdock [7] claimed that it was practical for men and women to specialize in heavy and light tasks respectively based on their hormonal differences and build. Men produce greater levels of testosterone and are physically bigger than women making then better suited for manual labor.

For Oakley [6] while masculinity and femininity are universal constructs there are inter-cultural differences. She challenged Murdock's proclaimed universal law of the sexual division of labor by noting that among the Alorese of Indonesia it was women who performed many tasks such as lumbering, while men performed tasks such as basket weaving. In Alor basket weaving was regarded as masculine. This contrasts strikingly with Western culture where basket weaving is associated with femininity and lumbering with masculinity.

\section{Pioneer Research: A Description}

Pioneer research is any investigation which was first to examine any social problem, issue or phenomenon. Thus the goal is to provide sufficient opportunity for the topic to be explored in subsequent research endeavors. The scientist is free, as circumstances dictate, to utilize any means of developing his ground breaking theory. The analysis of numbers alone, words alone or a combination of these is possible.

Pioneering research is akin to the principle of "relativity" since at any given point we can never know everything about anything. Additionally, due to cultural differences between and within groups we cannot generalize about many things. For example, some Marxist-oriented writers explain differences in class achievement patterns by examining differences in values, life styles and speech patterns between the middle and lower classes. Bernstein [8] did this in his work on the "elaborated" and "restricted" codes of the middle class and working class respectively. 
In contemporary times it may be more challenging for sociologists and to act as pioneers because there is so much published information about social phenomena. However, despite this there are issues that can be investigated. For example in Trinidad and Tobago there is a cultural practice known as 'liming' where on evenings predominantly urban boys and young men "hang about" street corners usually passing the time doing almost nothing. If we could understand the meanings and motives behind this practice then we could gain a deeper appreciation of informal mechanisms people use for developing strong community bonds. Invariably they may also indicate much evidence similar to Young and Willmott's [9] study of Bethnal Green in which strong community and familial ties thrived in an urban setting.

\section{Complementary Research: A Description}

Complementary research is any attempt to take pioneer research farther. I suggest that the hypothetico-deductive method propounded by Popper [10] provides a good fit for this purpose. Instead of building a theory the research seeks to falsify the pioneer theory. Quantitative or qualitative data or a mixture (of them) may be used. More critically though the philosophy supported here is the discovery of 'complements' or additional pieces of information that would provide for a more comprehensive understanding of social life.

We must veer away from the traditional proclivity of criticizing other researchers on grounds that may be too insignificant. Interestingly though, I do not discount challenges based on limitations in methodology and theoretical conceptualization if they distort social reality. I advocate instead a realist position based on the principles of relativity, relevance and value. It is sufficient to say that we can never know the whole truth or find casual explanations of phenomena that are completely value neutral and wholly valid and reliable. The best we can do is to arrive at the closest estimate of these parameters within our findings.

Feminists who advocate standpoint epistemology are commended for recognizing the intricacies of knowledge. Hill Collins [11] advocates for the experiences of black women to get told more often so that the full extent of their triple discrimination (class, race and sex) can be fully understood. By upholding constructivist ideological paradigms they assist our understanding of knowledge as a subjective construct of the constructor. Both the researcher and the subject can work together to construct their views on social reality.

I propose a quasi post-modernist philosophy of uniqueness. This is an aggrandizement of its premise that research should explicate participant idiosyncrasies. However, it should depart from its diatribe about the uselessness of metanarratives propounded by Lyotard [12]. Instead I proffer that metanarratives aimed to provide depth in understanding behavior in its context be employed in social research.

\section{General Arguments about Research: Strengthening the Case for Pioneer Research and Complementary Research}

All research (pioneer or complementary) is useful regardless of its orientation. This is contingent, of course, upon the notion that its usefulness is borne out by its contribution to academia and/or enhancement of social life. Research is best assessed in terms of its intent. Therefore factors such as time, cost, interest, and theoretical appropriateness (to name a few) should guide the choice of method. A researcher short on time will opt for quantitative over qualitative data collection. Qualitative research can prove to be more costly than a survey which requires less financial resources for completion. No one would dispute the fact that anthropologists spending years in small group research projects find it more costly than researchers e-mailing survey questions. A researcher interested in understanding the way a group of drug addicts constructs knowledge of their addiction will turn to interviews as did Becker [13] in studying outsiders.

On the other hand sub-cultural theorists who sought to correlate delinquency to social class resorted to multivariate analysis of official statistics. Merton [14], Cohen [15], and Cloward and Ohlin [16] were proponents of sub-cultural influences on male delinquency. For purposes of simplicity it is being contested that quantitative data is better for testing theories and qualitative data for building theories. However, there is no deterrence from using either of them inductively or deductively. The custom of inductive-qualitative, deductive quantitative is neither universally natural nor convenient. Would it be wrong for an interviewer to research evaluating a social policy initiative to interview a small group of participants affected by a specific social problem? Would it be inappropriate for a theory to be induced using numerical data?

All social research is generic once it aims to elucidate components of human behavior. Research traditionally 
defined as quantitative must produce qualitative outcomes. Durkheim [3] demonstrated this when he concluded that integration and regulation were the causes of suicide. Conversantly, research traditionally defined as qualitative entails quantitative features, concerns and outcomes. For instance, some qualitative researchers count the number of times particular codes appear in the data. This is true of Lobban [17] who through content analysis counted (the disproportionate) number of times heroes and heroines were depicted in early reading schemes.

The overarching argument can be summed up by stating that fundamental ontological differences attributed to the terms quantitative and qualitative are at best spurious and superficial. All research projects combine statistics and qualities in different strengths and proportions. In any research project sampling, for example, is a quantitative facility. Every researcher declares the number of persons from whom data was gathered. In all research projects the discussion of findings is a description of quality. Raw numbers are inadequate for demonstrating the researcher's contribution to knowledge and the value of the knowledge in terms of its validity and reliability.

The term "mixed method" is misleading as it ignores the similarities between statistical and textual analyses. Mixed methods should be viewed as complete research capable of illuminating a phenomenon. Mixed research is truly generic and in the best position to support the principle of complementarity. The manner of mixing is irrelevant. Creswell [18] identified four forms of mixing; explanatory sequential, exploratory sequential, embedded and convergent. There is no need for us to examine them here as they are outside the scope of this paper.

The employment of a systematic approach to research for academic or policy prescriptions is desirable and should supersede emphasis on a quantitative-qualitative dichotomy. There is no research devoid of researcher values or free of bias. Neither quantitative nor qualitative data ensures complete value-freedom. Realist views of science emphasize this by suggesting that both natural and social scientists face problems with precision and objectivity/validity, Urry and Keat [19].

Features of research such as validity and reliability are difficult to attain and are mere estimates of quality. Therefore claims that the use of statistics is high in reliability and experiential research superior in terms of validity stretch our sociological and "research" imagination too far. In light of this assertion it is feasible to consider the worth of research in terms of its standpoint. This is an endorsement of the feminist position on standpoint epistemology. Researchers should be impelled to make public any biases in their research findings especially if they are directed toward policy evaluation and knowledge building.

\section{Approaches to Complementary Research}

There is no limit to the finding of complements. However, I suggest three major approaches. The first is criticism of theories, concepts, measures and procedures by testing a pioneer theory. Mere criticism is worthless if it fails to provide new insights into human behavior.

The second calls for a reversal of the relationship between well established dependent and independent variables. I have done this in studying the relationship between education (independent variable) and neighborhood climate (dependent variable) instead of examining them in the traditional manner: neighborhood $=$ independent variable; education $=$ dependent variable); Berkeley [20]. This suggests that the researcher purposively selects relationships between variables that have been traditionally measured in a well established manner and studies them in reverse order. This can be accomplished in a single research project (single reverse dependent study).

The third employs a process of double dependence in a single research project that enables two main variables to take turns being the dependent variable. The main objective is to compare the extent of causality/predictability of two or more factors within the same sample. For instance, if research proves that an independent variable accounts for a certain amount of variance in a dependent variable, it may not mean that if the position were reversed that the variance would be the same.

\section{Conclusions}

This paper sought to develop an alternative research classification (pioneer and complementary) to quantitative and qualitative. I contend that the terms pioneer and complementary are more appropriate for classifying research, Berkeley [20]. It argued a cogent and compelling case for the adoption of the terms pioneer and complementary. This would enable social scientists to evaluate research on grounds of their relevance to social progress. I propose a return to some aspects of the philosophy of Weber, cited in Gerth and Mills [21] which is often forgotten in modern sociological discourses. More specifically I refer to his writings which do not necessarily call for complete dismissal of statistical data but for a focus on verstehen (interpretive understanding). 
This, I believe, is the re-affirmation of complementarity in sociology. Giddens [22] must be credited for his attempt at theorizing structuration (duality of agency and structure) in congruence with the principle of complementarity.

The terms quantitative and qualitative are neither socially valuable nor adequately descriptive. They serve only to perpetuate distinctions which do nothing to advance human societies. Instead it is sagacious to perceive quantitative and qualitative as types of data, data gathering techniques and techniques of data analysis.

\section{References}

[1] Glaser, B.G. and Strauss, A.L. (1967) The Discovery of Grounded Theory: Strategies for Qualitative Research. Aldine Publishing Company, Chicago.

[2] Strauss, A.L. and Corbin, J.M. (2008) Basics of Qualitative Research: Techniques and Procedures for Developing Grounded Theory. Sage Publications, Los Angles.

[3] Durkheim, E. (1938) The Rules of Sociological Method. The Free Press, New York.

[4] Atkinson, J.M. (1978) Discovering Suicide: Studies in the Social Organization of Sudden Death. Macmillan, London.

[5] Garfinkel, H. (1984) Studies in Ethnomethodology. Polity Press, Cambridge.

[6] Oakley, A. (1974) The Sociology of Housework. Pantheon Books, New York.

[7] Murdock, G.P. (1949) Social Structure. The MacMillan Company, New York.

[8] Bernstein, B. (1971) Class, Codes and Control: Theoretical Studies towards a Sociology of Language. Routledge \& Kegan Paul, London. http://dx.doi.org/10.4324/9780203014035

[9] Young, M. and Willmott, P. (1973) The Symmetrical Family. Hamondsworth: Penguin.

[10] Popper, K. (2002) Conjectures and Refutations: The Growth of Scientific Knowledge. Routledge, London.

[11] Hill Collins, P. (2000) Black Feminist Thought: Knowledge, Consciousness, and the Politics of Empowerment. Routledge, New York.

[12] Lyotard, J.-F. (1979) Introduction: The Postmodern Condition: A Report on Knowledge. Manchester University Press, Manchester.

[13] Becker, H. (1963) Outsiders: Studies in the Sociology of Deviance. The Free Press, New York.

[14] Merton, R.K. (1957) Social Theory and Social Structure. Revised Edition, Free Press, New York.

[15] Cohen, A. (1955) Delinquent Boys. Free Press, New York.

[16] Cloward, R. and Ohlin, L. (1960) Delinquency and Opportunity. Free Press, New York.

[17] Creswell, J. (2003) Research Designs: Qualitative, Quantitative, and Mixed Methods Approaches. Sage, Thousand Oaks.

[18] Lobban, G. (1974) The Presentation of Sex Roles in British Reading Schemes. Forum, 16, 57-60.

[19] Urry, J. and Keat, R. (1975) Social Theory as Science. Routledge and Kegan Paul, London.

[20] Berkeley, B. (2009) "New” Directions in Social Science Research: The Development of Complementary Theory. Lambert Academic Publishing, Saarbrücken.

[21] Weber, M., Gerth, H.H. and Mills, C.W., Eds. (1946) From Max Weber: Essays in Sociology. Oxford University Press, New York.

[22] Giddens, A. (1986) Constitution of Society: Outline of the Theory of Structuration. University of California Press, Oakland. 